\title{
Film-Forming Characteristics of Grease in Point Contact Under Swaying Motions
}

\author{
Gang Li $\cdot$ Chenhui Zhang $\cdot$ Jianbin Luo • \\ Shuhai Liu $\cdot$ Guoxin Xie $\cdot$ Xinchun Lu
}

Published online: 16 May 2009

(C) Springer Science+Business Media, LLC 2009

\section{Erratum to: Tribol Lett \\ DOI 10.1007/s11249-009-9433-7}

While performing additional investigations, we have discovered some minor errors in Eqs. (2), (3), (11), and (12) in the original article. The corrected equations are as follows:

$h_{c}=1.899 \alpha^{0.53} \frac{\left(u \eta_{G}\right)^{0.67} R^{\prime 0.464}}{E^{\prime 0.073} W^{0.067}}$

$h_{\min }=1.791 \alpha^{0.49} \frac{\left(u \eta_{G}\right)^{0.68} R^{\prime 0.466}}{E^{0.117} W^{0.073}}$

$K_{1}=1.899 \alpha^{0.53} \frac{\eta_{G}^{0.67} R^{\prime 0.464}}{E^{\prime 0.073} W^{0.067}}$

$K_{2}=1.791 \alpha^{0.49} \frac{\eta_{G}^{0.68} R^{\prime 0.466}}{E^{0.117} W^{0.073}}$

In this case, the values in Fig. 10 decrease slightly. However, these errors do not affect the conclusion that at a same entering velocity, the central film thickness under deceleration is higher than that under acceleration. The corrected Fig. 10 is as follows:

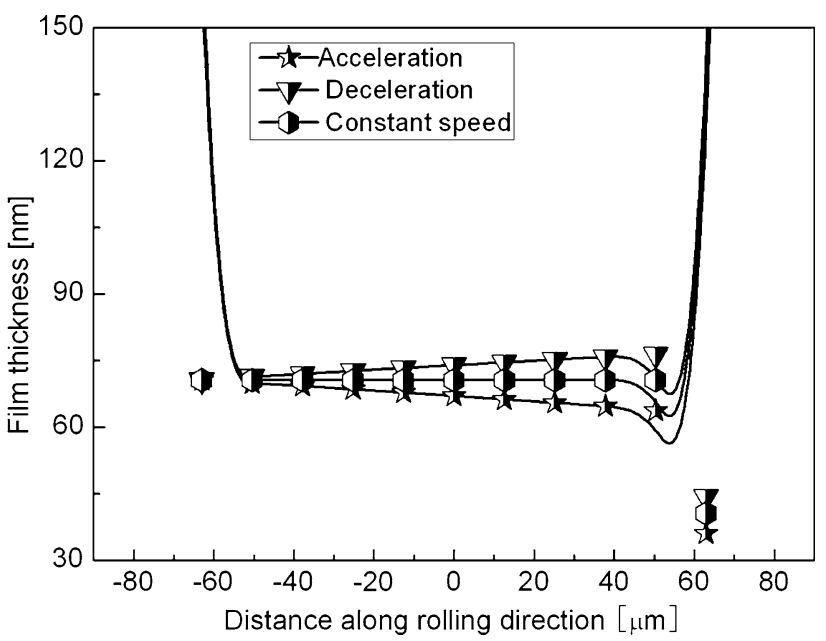

Fig. 10 Comparison of the film thickness profiles and film thickness values under different accelerations $(\mathrm{a}>0, \mathrm{a}<0$, and $\mathrm{a}=0)$

The online version of the original article can be found under doi: 10.1007/s11249-009-9433-7.

G. Li · C. Zhang $(\bowtie) \cdot$ J. Luo $\cdot$ S. Liu $\cdot$ G. Xie $\cdot$ X. Lu

State Key Laboratory of Tribology, Tsinghua University, Beijing 100084, China

e-mail: chzhang@tsinghua.edu.cn

G. Li

e-mail: ligang06@mails.tsinghua.edu.cn 